\title{
Anti-HER2/Anti-CD3 Bispecific Monoclonal Antibody BTRC 4017A
}

National Cancer Institute

\section{Source}

National Cancer Institute. Anti-HER2/Anti-CD3 Bispecific Monoclonal Antibody BTRC

4017A. NCl Thesaurus. Code C156705.

An anti-human epidermal growth factor receptor 2 (HER2)/anti-CD3 T-cell-dependent

bispecific (TDB) monoclonal antibody with potential immunostimulatory and

antineoplastic activities. Upon administration, anti-HER2/anti-CD3 bispecific monoclonal

antibody BTRC4017A possesses two antigen recognition sites, one for HER2, a tyrosine kinase receptor overexpressed by many cancer cell types, and one for the CD3 complex, a group of T-cell surface glycoproteins that interact with the T-cell receptor (TCR). Upon administration of BTRC4017A, this bispecific monoclonal antibody simultaneously binds to both CD3-expressing T-cells and HER2-expressing cancer cells, thereby crosslinking HER2-expressing tumor cells and cytotoxic T-lymphocytes (CT Ls). This may result in potent CTL-mediated lysis of HER2-expressing tumor cells. HER2 plays a key role in tumor cell proliferation and tumor vascularization. 